AMS JOURNAL STYLE

Volume 000, 0000

\title{
PROPERTIES OF IDEALS ON THE GENERALIZED CANTOR SPACES
}

\author{
JAN KRASZEWSKI
}

\begin{abstract}
We define a class of productive $\sigma$-ideals of subsets of the Cantor space $2^{\omega}$ and observe that both $\sigma$-ideals of meagre sets and of null sets are in this class. From every productive $\sigma$-ideal $\mathcal{J}$ we produce a $\sigma$-ideal $\mathcal{J}_{\kappa}$ of subsets of the generalized Cantor space $2^{\kappa}$. In particular, starting from meagre sets and null sets in $2^{\omega}$ we obtain meagre sets and null sets in $2^{\kappa}$, respectively. Then we investigate additivity, covering number, uniformity and cofinality of $\mathcal{J}_{\kappa}$. For example, we show that

$$
\operatorname{non}(\mathcal{J})=\operatorname{non}\left(\mathcal{J}_{\omega_{1}}\right)=\operatorname{non}\left(\mathcal{J}_{\omega_{2}}\right)
$$
\end{abstract}

Our results generalizes those from [5].

\section{INTRODUCTION}

In this paper we shall discuss the properties of canonical $\sigma$-ideals of subsets of generalized Cantor spaces $2^{\kappa}$, for example the $\sigma$-ideal of null sets and of meagre sets.

In the 80's several people investigated relations between the $\sigma$-ideal $\mathcal{N}$ of null subsets of the classical Cantor space $2^{\omega}$ and the $\sigma$-ideal $\mathcal{N}_{\kappa}$ of null subsets of the generalized Cantor space $2^{\kappa}$ for some uncountable cardinal number $\kappa$. One of the most important questions was what were the connections between cardinal coefficients (such as add, cov, non and cof) of $\mathcal{N}$ and these of $\mathcal{N}_{\kappa}$. The answer was given independently by Cichoń ([1], unpublished) and Fremlin ([5]). Both authors obtained almost the same results, except for two of them: Theorem 3.4 for null sets (only Fremlin) and Theorem 3.10 for null sets (only Cichoń).

A natural question arose whether measure-theoretic tools were really necessary to get these results. In this paper we give a complete answer to it. In order to do this we extract the combinatorial principles that are considered by both authors and show that similar results to those which were obtained by them can be proved for a much wider class of ideals.

In the first section we formulate a notion of productivity. If we identify $2^{\omega}$ with its square using canonical homeomorphism then we can say a bit imprecisely that an ideal $\mathcal{J}$ of subsets of $2^{\omega}$ is productive if for every $A \in \mathcal{J}$ the cylinder $A \times 2^{\omega}$ is in $\mathcal{J}$. We observe that $\sigma$-ideals of meagre sets and of null sets are productive.

Received by the editors.

Key words and phrases. Cantor space, $\sigma$-ideals, null sets, meagre sets, cardinal functions. 
Moreover, there exists the least non-trivial productive $\sigma$-ideal on $2^{\omega}$ called $\mathcal{S}_{2}$. Then we describe a method of constructing a $\sigma$-ideal $\mathcal{J}_{\kappa}$ of subsets of $2^{\kappa}$ from a given productive $\sigma$-ideal $\mathcal{J}$ of subsets of $2^{\omega}$. This method is reasonable because starting from meagre sets (null sets) in $2^{\omega}$ we obtain meagre sets (resp. null sets) in $2^{\kappa}$ and it is our main tool throughout this paper.

In the next section we completely describe the additivity and (with some additional weak assumption) cofinality of $\mathcal{J}_{\kappa}$. The third section is devoted to the uniformity of $\mathcal{J}_{\kappa}$. The most surprising result is that for every productive $\sigma$-ideal $\mathcal{J}$ we have

$$
\operatorname{non}(\mathcal{J})=\operatorname{non}\left(\mathcal{J}_{\omega_{1}}\right)=\operatorname{non}\left(\mathcal{J}_{\omega_{2}}\right)
$$

(for meagre sets it was proved by Veličkovič and remained unpublished). Moreover, uniformities of $\mathcal{J}_{\kappa}$ 's form an increasing sequence. However, it is possible to find bounds for $\operatorname{non}\left(\mathcal{J}_{\kappa}\right)$ which depend only on $\kappa$, not on $\mathcal{J}$ :

$$
\log (\kappa) \leq \operatorname{non}\left(\mathcal{J}_{\kappa}\right) \leq(\log (\kappa))^{\omega}
$$

We also prove Theorem 3.9, which is a powerful tool for showing several absolute inequalities concerning non's as well as some consistency results.

In the fourth section we deal with the covering number of $\mathcal{J}_{\kappa}$. These results are quite different from the previous ones as covering numbers of $\mathcal{J}_{\kappa}$ 's form a decreasing sequence. We prove that this sequence stabilizes for some cardinal number $\kappa \leq \mathfrak{c}$. In the last section we consider several models of Set Theory. In particular, we compute all cardinal coefficients of $\mathcal{J}_{\kappa}$ assuming Generalized Continuum Hypothesis and investigate a function $F(\kappa)=\operatorname{cov}\left(\mathcal{M}_{\kappa}\right)$ (where $\mathcal{M}$ stands for the $\sigma$-ideal of meagre sets) in some models of set theory obtained by the method of forcing.

The paper contains the main part of the author's Ph.D. thesis. The author would like to thank Professor J. Cichon for his help during the preparation of this paper and Professor D. H. Fremlin for his fruitful remarks.

\section{BASIC DEFINITIONS AND FACTS}

In this paper we deal with the generalized Cantor space $2^{\kappa}$ interpreted as the set of all functions from an infinite cardinal number $\kappa$ into the set $\{0,1\}$. This spaces are endowed with the standard product topology. Moreover, we consider the standard product measure on $2^{\kappa}$.

We use standard set-theoretical notation and terminology derived from [12]. Let us remind that the cardinality of the set of all real numbers is denoted by $c$. The cardinality of a set $X$ is denoted by $|X|$. If $\kappa$ is a cardinal number then $[X]^{\kappa}\left([X]^{\leq \kappa}\right)$ denotes the family of all subsets of the set $X$ of cardinality $\kappa$ (not greater than $\kappa$, respectively). $X^{<\omega}$ denotes the set of all finite sequences of elements of the set $X$. If $\varphi: X \rightarrow Y$ is a function then $\operatorname{rng}(\varphi)$ denotes the range of $\varphi$. If $A \subseteq Y$ then $\varphi^{-1}[A]$ denotes the pre-image of $A$.

The $\sigma$-ideals of meagre subsets and of null subsets of $2^{\omega}$ are denoted by $\mathcal{M}$ and $\mathcal{N}$, respectively.

In addition, we introduce some extra notation in order to simplify further considerations. Let $\lambda$ and $\kappa$ be any infinite cardinal numbers. We put $\operatorname{Inj}(\lambda, \kappa)=$ $\left\{\varphi \in \kappa^{\lambda}: \varphi\right.$ is an injection $\}$. 
Definition. For $A \subseteq 2^{\kappa}$ and $\varphi \in \operatorname{Inj}(\omega, \kappa)$ we put $\varphi * A=\{x \circ \varphi: x \in A\}$. For $B \subseteq 2^{\omega}$ and $\varphi \in \operatorname{Inj}(\omega, \kappa)$ we put $B_{\varphi}=\left\{x \in 2^{\kappa}: x \circ \varphi \in B\right\}$.

It is reasonable to add some intuitions to this definition. Namely, if $T$ is a subset of $\kappa$ then we identify the generalized Cantor space $2^{\kappa}$ and the space $2^{T} \times 2^{\kappa \backslash T}$ using the canonical homeomorphism $\pi_{T}$ defined by $\pi_{T}(x)=(x\lceil T, x \uparrow(\kappa \backslash T))$. Then, if we treat $2^{\kappa}$ as a product $2^{\operatorname{rng}(\varphi)} \times 2^{\kappa \backslash \operatorname{nng}(\varphi)}$ for some $\varphi \in \operatorname{Inj}(\omega, \kappa)$ and canonically identify $2^{\text {rng }(\varphi)}$ with $2^{\omega}$ using $\varphi$ then for any set $A \subseteq 2^{\kappa}$ we can interpret the set $\varphi * A$ as a projection of $A$ into $2^{\operatorname{rng}(\varphi)}$. Similarly, for any set $B \subseteq 2^{\omega}$ we can treat the set $B_{\varphi}$ as a cylinder $B \times 2^{\kappa \backslash \operatorname{rng}(\varphi)}$.

Obviously, $\varphi * A \subseteq 2^{\omega}$ and $B_{\varphi} \subseteq 2^{\kappa}$. Another simple observation is that for $B \subseteq 2^{\omega}$ and $\varphi \in \operatorname{Inj}(\omega, \kappa)$ we have $\varphi * B_{\varphi}=B$. Similarly, for $A \subseteq 2^{\kappa}$ and $\varphi \in \operatorname{Inj}(\omega, \kappa)$ we have $A \subseteq(\varphi * A)_{\varphi}$.

From now on let $\mathcal{J}$ be any $\sigma$-ideal of subsets of $2^{\omega}$ and $\kappa$ be any infinite cardinal number. We define

$$
\kappa(\mathcal{J})=\left\{A \subseteq 2^{\kappa}:(\exists \varphi \in \operatorname{Inj}(\omega, \kappa)) \varphi * A \in \mathcal{J}\right\}
$$

If $A \subseteq 2^{\kappa}$ then any $\varphi \in \operatorname{Inj}(\omega, \kappa)$ such that $\varphi * A \in \mathcal{J}$ we called a witness for $A$. If $\mathcal{I} \subseteq \mathcal{J}$ then $\kappa(\mathcal{I}) \subseteq \kappa(\mathcal{J})$. Moreover, we have $\mathcal{J} \subseteq \omega(\mathcal{J})$, because for every $A \in \mathcal{J}$ the identity on $\omega$ is a witness for $A$. The $\sigma$-ideal generated by the family $\kappa(\mathcal{J})$ we denote by $\mathcal{J}_{\kappa}$.

Definition. The ideal $\mathcal{J}$ is productive if $\omega(\mathcal{J}) \subseteq \mathcal{J}$.

We formulate some equivalent versions of this property now.

Fact 1.1. For any $\sigma$-ideal $\mathcal{J}$ of subsets of $2^{\omega}$ the following conditions are equivalent:

(a) $\mathcal{J}$ is productive,

(b) $\left(\forall A \subseteq 2^{\omega}\right)(\forall \varphi \in \operatorname{Inj}(\omega, \omega))(\varphi * A \in \mathcal{J} \Rightarrow A \in \mathcal{J})$,

(c) $\left(\forall A \subseteq 2^{\omega}\right)(\forall \varphi \in \operatorname{Inj}(\omega, \omega))\left(A \in \mathcal{J} \Rightarrow A_{\varphi} \in \mathcal{J}\right)$.

Proof. The equivalence of conditions (a) and (b) follows directly from the definition of the productivity of $\mathcal{J}$. Suppose now that (b) holds and let $A \in \mathcal{J}$ and $\varphi \in$ $\operatorname{Inj}(\omega, \omega)$. As $\varphi * A_{\varphi}=A \in \mathcal{J}$ then according to (b) we have $A_{\varphi} \in \mathcal{J}$ and, consequently, (c) holds as well.

On the other hand, let us assume (c) and take $A \subseteq 2^{\omega}$ and $\varphi \in \operatorname{Inj}(\omega, \omega)$ such that $\varphi * A \in \mathcal{J}$. Then (c) gives us that $(\varphi * A)_{\varphi} \in \mathcal{J}$. But as we know $A \subseteq(\varphi * A)_{\varphi}$ so $A \in \mathcal{J}$. Hence (b) is true.

We can intuitively interpret the definition of productivity in such a way that justifies its name. Namely, thanks to Fact 1.1 and the previous description, we can say that $\mathcal{J}$ is productive if for every $\varphi \in \operatorname{Inj}(\omega, \omega)$ and every set $A \subseteq 2^{\operatorname{rng}(\varphi)}$ if $A$ is in $\mathcal{J}$ then the cylinder $A \times 2^{\omega \backslash \operatorname{rng}(\varphi)}$ is in $\mathcal{J}$.

Directly from their definitions we deduce that the $\sigma$-ideals of meagre subsets and of null subsets of $2^{\omega}$ are productive. Also the well-known $\sigma$-ideal generated by closed null subsets of $2^{\omega}$ is productive.

Not every $\sigma$-ideal of subsets of $2^{\omega}$ is productive. However, every $\sigma$-ideal has its "productive closure". 
Fact 1.2. For any $\sigma-i$ deal $\mathcal{J}$ of subsets of $2^{\omega}$ the $\sigma-i d e a l \mathcal{J}_{\omega}$ is productive.

Proof. It is enough to prove that $\omega\left(\mathcal{J}_{\omega}\right) \subseteq \mathcal{J}_{\omega}$. Let $A \subseteq 2^{\omega}$ be a member of $\omega\left(\mathcal{J}_{\omega}\right)$ and let $\varphi \in \operatorname{Inj}(\omega, \omega)$ be its witness. It means that $\varphi * A \in \mathcal{J}_{\omega}$ which implies $\varphi * A \subseteq \bigcup_{i<\omega} A_{i}$ for some family $\left\{A_{i}: i<\omega\right\} \subseteq \omega(\mathcal{J})$. Thus

$$
A \subseteq(\varphi * A)_{\varphi} \subseteq\left(\bigcup_{i<\omega} A_{i}\right)_{\varphi}=\bigcup_{i<\omega}\left(A_{i}\right)_{\varphi}
$$

Let $\varphi_{i} \in \operatorname{Inj}(\omega, \omega)$ be a witness for $A_{i}$ for each $i<\omega$. To finish the proof we need the following simple observation:

Claim. For every $A \subseteq 2^{\omega}$ and $\varphi, \psi \in \operatorname{Inj}(\omega, \omega)$ we have $\psi *(\varphi * A)=(\varphi \circ \psi) * A$.

Hence for each $i<\omega$ we have

$$
\left(\varphi \circ \varphi_{i}\right) *\left(A_{i}\right)_{\varphi}=\varphi_{i} *\left(\varphi *\left(A_{i}\right)_{\varphi}\right)=\varphi_{i} * A_{i} \in \mathcal{J}
$$

so $\left(A_{i}\right)_{\varphi}$ is a member of $\omega(\mathcal{J})$ and $\varphi \circ \varphi_{i}$ is its witness. Consequently, $\bigcup_{i<\omega}\left(A_{i}\right)_{\varphi}$ is a member of $\mathcal{J}_{\omega}$ and so is $A$.

The $\sigma$-ideal $\left[2^{\omega}\right]^{\leq \omega}$ of all countable subsets of $2^{\omega}$ is not productive. The $\sigma$-ideal $\left(\left[2^{\omega}\right] \leq \omega\right)_{\omega}$ is the least non-trivial productive $\sigma$-ideal of subsets of the Cantor space. It is denoted by $\mathcal{S}_{2}$ and was investigated in [3]. Earlier it appeared in [13], but only incidentally. It occurred that properties of this $\sigma$-ideal are strongly connected with some intensively studied combinatorial properties of subsets of natural numbers (the splitting and reaping numbers). For example, non $\left(\mathcal{S}_{2}\right)=\aleph_{0-\mathfrak{s}}$ (see [11] for the definition of $\aleph_{0-\mathfrak{s}}$ and more discussion).

It is worth noting that the $\sigma$-ideal $\mathcal{J}_{\omega}$ is not necessarily proper even if $\mathcal{J}$ is proper. For example, if $\mathcal{J}$ is a $\sigma$-ideal orthogonal to $\mathcal{S}_{2}$ (i.e. there exist sets $A \in \mathcal{J}$ and $B \in \mathcal{S}_{2}$ such that $A \cup B=2^{\omega}$ ) then $\mathcal{J}_{\omega}$ must be improper because due to Fact 1.2 it is productive and thus contains $\mathcal{S}_{2}$.

The following fact holds:

Fact 1.3. If $\mathcal{J}$ is productive then $\kappa(\mathcal{J})=\mathcal{J}_{\kappa}$ for any infinite cardinal number $\kappa$.

Proof. We left easy calculations to the reader.

Throughout this paper we will investigate $\sigma$-ideals $\mathcal{J}_{\kappa}$ for a certain productive $\sigma$-ideal $\mathcal{J}$ of subsets of $2^{\omega}$. First, we have to make sure that it will not be art for art sake, namely if we consider the $\sigma$-ideal $\mathcal{M}$ of meagre subsets of $2^{\omega}$ or the $\sigma$-ideal $\mathcal{N}$ of null subsets of $2^{\omega}$ then $\mathcal{M}_{\kappa}$ and $\mathcal{N}_{\kappa}$ will be the $\sigma$-ideals of meagre or null subsets of $2^{\kappa}$, respectively.

Fact 1.4. $\mathcal{M}_{\kappa}$ is the $\sigma$-ideal of meagre subsets of $2^{\kappa}$ in the standard product topology. $\mathcal{N}_{\kappa}$ is the $\sigma$-ideal of null subsets of $2^{\kappa}$ in the standard product measure.

Proof. 1. The meagre case.

In order to show that every element of $\mathcal{M}_{\kappa}$ is meagre it is enough to observe that for every nowhere dense subset $E$ of $2^{\omega}$ and every $\varphi \in \operatorname{Inj}(\omega, \kappa)$ the set $E_{\varphi}$ is nowhere dense in $2^{\kappa}$. 
To complete the proof it is enough to show that every closed nowhere dense subset $D$ of $2^{\kappa}$ is in $\mathcal{M}_{\kappa}$. Let us remind that if $\sigma$ is a finite partial function from $\kappa$ into the set $\{0,1\}$ then the set

$$
[\sigma]_{\kappa}=\left\{x \in 2^{\kappa}: \sigma \subseteq x\right\}
$$

is open and the family of all subsets of $2^{\kappa}$ of this type is a basis for the standard product topology.

Let $\left\{\left[\sigma_{i}\right]_{\kappa}: i \in I\right\}$ be a maximal family of pairwise disjoint open basis subsets of $2^{\kappa}$ which are disjoint with the set $D$ as well. The set $I$ is at most countable due to countable chain condition of the space $2^{\kappa}$. Thus there exists an injection $\varphi \in$ $\operatorname{Inj}(\omega, \kappa)$ such that $\bigcup_{i<\omega} \operatorname{dom}\left(\sigma_{i}\right) \subseteq \operatorname{rng}(\varphi)$. Then $\sigma_{i} \circ \varphi$ is a partial function from $\omega$ into the set $\{0,1\}$ and $\varphi *\left[\sigma_{i}\right]_{\kappa}=\left[\sigma_{i} \circ \varphi\right]_{\omega}$ for each $i \in I$. Then $\left\{\left[\sigma_{i} \circ \varphi\right]_{\omega}: i \in I\right\}$ is a maximal family of pairwise disjoint open basis subsets of $2^{\omega}$ which are disjoint with the set $\varphi * D$. Consequently, the set $\varphi * D$ is a nowhere dense subset of $2^{\omega}$, which ends the proof.

2. The null case

Let $\mu_{\kappa}$ denotes standard product measure on $2^{\kappa}$. To begin with, let us observe that for every basis open set $B \subseteq 2^{\omega}$ of the form mentioned above and every $\varphi \in \operatorname{Inj}(\omega, \kappa)$ we have $\mu_{\omega}(B)=\mu_{\kappa}\left(B_{\varphi}\right)$. Thus straight from the definition of a product measure we can deduce that every set from $\mathcal{N}_{\kappa}$ is null.

Conversely, let $A$ be a null subset of $2^{\kappa}$. For every natural number $n$ there exists a family $\left\{\sigma_{i}^{n}: i<\omega\right\}$ of finite partial functions from $\kappa$ into the set $\{0,1\}$ such that

$$
A \subseteq \bigcup_{i<\omega}\left[\sigma_{i}^{n}\right]_{\kappa} \quad \text { and } \quad \sum_{i<\omega} \frac{1}{2^{\left|\sigma_{i}^{n}\right|}}<\frac{1}{n+1}
$$

Let $\varphi \in \operatorname{Inj}(\omega, \kappa)$ be such that $\bigcup_{i, n<\omega} \operatorname{dom}\left(\sigma_{i}^{n}\right) \subseteq \operatorname{rng}(\varphi)$. Then for every $n \in \omega$ we have $\varphi * A \subseteq \bigcup_{i<\omega}\left[\sigma_{i}^{n} \circ \varphi\right]_{\omega}$. But

$$
\sum_{i<\omega} \frac{1}{2^{\left|\sigma_{i}^{n} \circ \varphi\right|}}=\sum_{i<\omega} \frac{1}{2^{\left|\sigma_{i}^{n}\right|}}<\frac{1}{n+1}
$$

Thus $\varphi * A$ is a null subset of $2^{\omega}$, so $A \in \mathcal{N}_{\kappa}$.

We shall use in our further considerations the following simple lemma.

Lemma 1.5. Let $\mathcal{J}$ be productive. If $A \in \mathcal{J}_{\kappa}$ and $\varphi \in \operatorname{Inj}(\omega, \kappa)$ is its witness then every $\psi \in \operatorname{Inj}(\omega, \kappa)$ such that $\operatorname{rng}(\varphi) \subseteq \operatorname{rng}(\psi)$ is also a witness for $A$.

Proof. Let $\eta=\psi^{-1} \circ \varphi$. Then $\eta \in \operatorname{Inj}(\omega, \omega)$ and $\eta *(\psi * A)=\varphi * A \in \mathcal{J}$. Thus $\psi * A \in \omega(\mathcal{J})=\mathcal{J}$.

Suppose that $\mathcal{J}$ is an ideal of subsets of $X$. We say that a family $\mathcal{B} \subseteq \mathcal{J}$ is cofinal with $\mathcal{J}$ if for each $A \in \mathcal{J}$ there exists such $B \in \mathcal{B}$ that $A \subseteq B$. We also call such a family $\mathcal{B}$ a base of $\mathcal{J}$. 
For an ideal $\mathcal{J}$ of subsets of the set $X$ we consider the following cardinal numbers

$$
\begin{aligned}
\operatorname{add}(\mathcal{J}) & =\min \{|\mathcal{A}|: \mathcal{A} \subseteq \mathcal{J} \& \bigcup \mathcal{A} \notin \mathcal{J}\} \\
\operatorname{cov}(\mathcal{J}) & =\min \{|\mathcal{A}|: \mathcal{A} \subseteq \mathcal{J} \& \bigcup \mathcal{A}=X\} \\
\operatorname{non}(\mathcal{J}) & =\min \{|B|: B \subseteq X \quad \& B \notin \mathcal{J}\} \\
\operatorname{cof}(\mathcal{J}) & =\min \{|\mathcal{B}|: \mathcal{B} \subseteq \mathcal{J} \& \mathcal{B} \text { is cofinal with } \mathcal{J}\}
\end{aligned}
$$

They are called the additivity, the covering number, the uniformity and the cofinality of $\mathcal{J}$, respectively. Note that if $\mathcal{J}$ is a proper ideal containing all points (i.e. $\cup \mathcal{J}=X$ ) then the following relations hold:

$$
\operatorname{add}(\mathcal{J}) \leq \operatorname{cov}(\mathcal{J}), \operatorname{add}(\mathcal{J}) \leq \operatorname{non}(\mathcal{J}), \operatorname{cov}(\mathcal{J}) \leq \operatorname{cof}(\mathcal{J}), \operatorname{non}(\mathcal{J}) \leq \operatorname{cof}(\mathcal{J})
$$

Moreover, $\operatorname{add}(\mathcal{J})$ is regular and $\operatorname{add}(\mathcal{J}) \leq \min \{\operatorname{cf}(\operatorname{non}(\mathcal{J})), \operatorname{cf}(\operatorname{cof}(\mathcal{J}))\}$.

There is a cardinal function which plays a big role in our further considerations, namely the cofinality of the $\sigma$-ideal of all countable subsets of $\kappa$. We denote it by $c_{\kappa}$ and remind some of its properties:

\section{Fact 1.6.}

(a) $\kappa \leq \lambda \Rightarrow c_{\kappa} \leq c_{\lambda}$;

(b) $\operatorname{cf}(\kappa)>\omega \Rightarrow c_{\kappa}=\max \left\{\kappa, \sup _{\omega \leq \xi<\kappa} c_{\xi}\right\}$;

(c) $\omega=\operatorname{cf}(\kappa)<\kappa \Rightarrow c_{\kappa}>\kappa$;

(d) $\omega_{1} \leq \kappa<\omega_{\omega} \Rightarrow c_{\kappa}=\kappa$;

(e) $\kappa \geq \mathfrak{c} \Rightarrow c_{\kappa}=\kappa^{\omega}$.

Proof. The proof of this fact can be found in [5] (see Theorem 6.17 (c)).

\section{Additivity And COFinality of $\mathcal{J}_{\kappa}$}

From now on we assume that $\mathcal{J}$ is a proper and productive $\sigma$-ideal of subsets of $2^{\omega}$ containing all points (i.e. $\bigcup \mathcal{J}=2^{\omega}$ ) and that $\kappa \geq \omega_{1}$.

Cardinal coefficients add, cov, non and cof of a $\sigma$-ideal $\mathcal{J}$ have a strong influence on those of $\mathcal{J}_{\kappa}$. However, the additivity of $\mathcal{J}_{\kappa}$ is absolute.

Theorem 2.1. $\operatorname{add}\left(\mathcal{J}_{\kappa}\right)=\omega_{1}$

Proof. For every ordinal number $\alpha<\kappa$ we define $I_{\alpha}=\{\beta<\kappa: \alpha \cdot \omega \leq \beta<\alpha \cdot \omega+\omega\}$ and $F_{\alpha}=\left\{x \in 2^{\kappa}: x \uparrow I_{\alpha} \equiv 0\right\}$. Obviously, $F_{\alpha} \in \mathcal{J}_{\kappa}$ for each $\alpha<\kappa$. Let $T$ be any uncountable subset of $\kappa$. Then

$$
\bigcup_{\alpha \in T} F_{\alpha} \notin \mathcal{J}_{\kappa}
$$

Actually, if there exists a witness $\varphi$ for $\bigcup_{\alpha \in T} F_{\alpha}$ then $\varphi * F_{\alpha} \in \mathcal{J}$ for every $\alpha \in T$. But there is $\alpha_{0} \in T$ such that $\operatorname{rng}(\varphi) \cap I_{\alpha_{0}}=\emptyset$ and, consequently, $\varphi * F_{\alpha_{0}}=2^{\omega}$ which is a contradiction.

Next results give us a precise description of the cofinality of described ideals. 
Theorem 2.2. $\operatorname{cof}\left(\mathcal{J}_{\kappa}\right) \leq \max \left\{\operatorname{cof}(\mathcal{J}), c_{\kappa}\right\}$.

Proof. Let $\left\{B_{\xi}: \xi<\lambda\right\} \subseteq \mathcal{J}$ be a base of $\mathcal{J}$ and let $\left\{\varphi_{\alpha}: \alpha<\eta\right\} \subseteq \operatorname{Inj}(\omega, \kappa)$ be a family such that $\left\{\operatorname{rng}\left(\varphi_{\alpha}\right): \alpha<\eta\right\}$ is a base of the $\sigma$-ideal of all countable subsets of $\kappa$. We define

$$
\mathcal{B}=\left\{\left(B_{\xi}\right)_{\varphi_{\alpha}}: \xi<\lambda, \alpha<\eta\right\}
$$

It is enough to show that $\mathcal{B}$ is a base of $\mathcal{J}_{\kappa}$.

Let $A \in \mathcal{J}_{\kappa}$ and let $\varphi$ be its witness. Thanks to the Lemma 1.5 we can assume that $\varphi=\varphi_{\alpha_{0}}$ for some $\alpha_{0}<\eta$. Let $Z=\varphi_{\alpha_{0}} * A$. We have $Z \in \mathcal{J}$ and it is easy to observe that if $Z \subseteq B_{\xi_{0}}$ for some $\xi_{0}<\lambda$ then $A \subseteq\left(B_{\xi_{0}}\right)_{\varphi_{\alpha_{0}}} \in \mathcal{B}$ which ends the proof.

Theorem 2.3. $\operatorname{cof}\left(\mathcal{J}_{\kappa}\right) \geq c_{\kappa}$.

Proof. We define the family $\left\{F_{\alpha}: \alpha<\omega_{1}\right\}$ in the same way as in the proof of Theorem 2.1. For every $B \subseteq 2^{\kappa}$ we put

$$
F_{B}=\left\{\alpha: F_{\alpha} \subseteq B\right\}
$$

We have already noticed that if $B \in \mathcal{J}_{\kappa}$ then $F_{B}$ is countable. Let $\left\{B_{\xi}: \xi<\lambda\right\} \subseteq$ $\mathcal{J}_{\kappa}$ be a base of $\mathcal{J}_{\kappa}$. We define

$$
\mathcal{B}=\left\{F_{B_{\xi}}: \xi<\lambda\right\}
$$

Let $T$ be any countable subset of $\kappa$. Then $\bigcup_{\alpha \in T} F_{\alpha} \subseteq B_{\xi_{0}}$ for some $\xi_{0}<\lambda$. Hence $T \subseteq F_{B_{\xi_{0}}} \in \mathcal{B}$.

To complete the characterization of the cofinality we have to introduce an additional property called the Weak Fubini Property (WFP).

Definition. The ideal $\mathcal{J}$ of subsets of $2^{\omega}$ has $W F P$ if for every $\varphi \in \operatorname{Inj}(\omega, \omega)$ and every $A \subseteq 2^{\omega}$ if $A_{\varphi}$ is in $\mathcal{J}$ then so is $A$.

The name can be explained by formulating the definition in terms of products, in a way that has been used before to explain the meaning of productivity. Namely, $\mathcal{J}$ has WFP if for every $\varphi \in \operatorname{Inj}(\omega, \omega)$ and every $A \subseteq 2^{\operatorname{rng}(\varphi)}$ if the cylinder $A \times 2^{\omega \backslash \operatorname{rng}(\varphi)}$ is in $\mathcal{J}$ then its projection into $2^{\operatorname{rng}(\varphi)}$, that is $A$, is also in $\mathcal{J}$ (after identifying $2^{\operatorname{rng}(\varphi)}$ with $2^{\omega}$ using $\varphi$ ). The $\sigma$-ideals mentioned previously, i.e. $\sigma-$ ideals of meagre sets and of null sets of $2^{\omega}, \sigma$-ideal generated by closed null subsets of $2^{\omega}$ and $\mathcal{S}_{2}$ obviously have WFP.

We will need the following technical lemma.

Lemma 2.4. If $\mathcal{J}$ is a productive ideal of subsets of $2^{\omega}$ having WFP then for every $\varphi \in \operatorname{Inj}(\omega, \kappa)$ and every $A \subseteq 2^{\omega}$ if $A_{\varphi} \in \mathcal{J}_{\kappa}$ then $A \in \mathcal{J}$.

Proof. We fix $\varphi \in \operatorname{Inj}(\omega, \kappa)$ and $A \subseteq 2^{\omega}$ such that $A_{\varphi} \in \mathcal{J}_{\kappa}$. Let $\psi$ be a witness for $A_{\varphi}$. We can assume that $\operatorname{rng}(\varphi) \subseteq \operatorname{rng}(\psi)$. Then $\eta=\psi^{-1} \circ \varphi$ is a member of $\operatorname{Inj}(\omega, \omega)$. A routine calculation shows that $A_{\eta} \subseteq \psi * A_{\varphi} \in \mathcal{J}$ so we can apply WFP to $\eta$ and $A$ and obtain the needed result. 
Theorem 2.5. If $\mathcal{J}$ has WFP then $\operatorname{cof}\left(\mathcal{J}_{\kappa}\right) \geq \operatorname{cof}(\mathcal{J})$.

Proof. Notice that the identity function on $\omega$ (denoted by $i d_{\omega}$ ) is a member of $\operatorname{Inj}(\omega, \kappa)$. For a set $B \subseteq 2^{\kappa}$ we construct a set

$$
H_{B}=\left\{x \in 2^{\omega}:\{x\}_{i d_{\omega}} \subseteq B\right\}
$$

We observe that $\left(H_{B}\right)_{i d_{\omega}}=\bigcup_{x \in H_{B}}\{x\}_{i d_{\omega}} \subseteq B$.

Let $\left\{B_{\xi}: \xi<\lambda\right\} \subseteq \mathcal{J}_{\kappa}$ be a base of $\mathcal{J}_{\kappa}$. We define a family

$$
\mathcal{B}=\left\{H_{B_{\xi}}: \xi<\lambda\right\}
$$

For every $\xi<\lambda$ we know that $\left(H_{B_{\xi}}\right)_{i d_{\omega}} \in \mathcal{J}_{\kappa}$. Applying the Lemma 2.4 to $i d_{\omega}$ and the set $H_{B_{\xi}}$ leads us to the conclusion that $H_{B_{\xi}} \in \mathcal{J}$.

Now fix any $A \in \mathcal{J}$. Then the set $A_{i d_{\omega}}$ is in $\mathcal{J}_{\kappa}$ so it is contained in $B_{\xi_{0}}$ for some $\xi_{0}<\lambda$. But this imply that $A \subseteq H_{B_{\xi_{0}}}$. Thus $\mathcal{B}$ is a base for $\mathcal{J}$.

Corollary 2.6. If $\mathcal{J}$ has WFP then $\operatorname{cof}\left(\mathcal{J}_{\kappa}\right)=\max \left\{\operatorname{cof}(\mathcal{J}), c_{\kappa}\right\}$.

It is worth observing that Theorems 2.2 and 2.3 do not need an assumption that $\mathcal{J}$ has WFP. The natural question is if Theorem 2.5 (and therefore Corollary 2.6) are true without any extra assumptions.

Question. Is it true that for any productive $\sigma$-ideal $\mathcal{J}$ of subsets of $2^{\omega}$ we have $\operatorname{cof}\left(\mathcal{J}_{\kappa}\right)=\max \left\{\operatorname{cof}(\mathcal{J}), c_{\kappa}\right\} ?$

\section{UNIFORMITY OF $\mathcal{J}_{\kappa}$}

The uniformity of $\mathcal{J}_{\kappa}$ differs from two coefficients considered in the previous section because no straightforward formula for it was found. Nevertheless, we can prove a series of useful inequalities. The first well-known fact shows that $\operatorname{non}\left(\mathcal{J}_{\kappa}\right)$ form an increasing sequence.

Fact 3.1. If $\lambda \leq \kappa$ then $\operatorname{non}\left(\mathcal{J}_{\lambda}\right) \leq \operatorname{non}\left(\mathcal{J}_{\kappa}\right)$.

Proof. Let $A$ be a subset of $2^{\kappa}$ which is not in $\mathcal{J}_{\kappa}$. We define a subset of $2^{\lambda}$ in the following way

$$
A^{\prime}=\{x\lceil\lambda: x \in A\} .
$$

It is enough to notice that if $\varphi \in \operatorname{Inj}(\omega, \lambda)$ would be a witness for $A^{\prime}$ then it would be a witness for $A$ as well (as a member of $\operatorname{Inj}(\omega, \kappa)$ ). Consequently, $A^{\prime}$ is not in $\mathcal{J}_{\lambda}$.

Theorem 3.2. $\operatorname{non}\left(\mathcal{J}_{\kappa}\right) \leq \max \left\{\operatorname{non}(\mathcal{J}), c_{\kappa}\right\}$.

Proof. Let $A \subseteq 2^{\omega}$ does not belong to $\mathcal{J}$ and let $\left\{\varphi_{\alpha}: \alpha<\eta\right\} \subseteq \operatorname{Inj}(\omega, \kappa)$ be a family such that $\left\{\operatorname{rng}\left(\varphi_{\alpha}\right): \alpha<\eta\right\}$ is a base of the $\sigma$-ideal of all countable subsets of $\kappa$. For every $\alpha<\eta$ we define a set

$$
\left.A_{\alpha}=\left\{x \in 2^{\kappa}: x \circ \varphi_{\alpha} \in A \quad \& \quad x \uparrow\left(\kappa \backslash \operatorname{rng}\left(\varphi_{\alpha}\right)\right) \equiv 0\right)\right\}
$$

Let $A^{*}=\bigcup_{\alpha<\eta} A_{\alpha}$. We will prove that $A^{*} \notin \mathcal{J}_{\kappa}$. 
Suppose otherwise and let $\varphi \in \operatorname{Inj}(\omega, \kappa)$ be a witness for $A^{*}$. As in the proof of Theorem 2.2 we can assume that $\varphi=\varphi_{\alpha_{0}}$ for some $\alpha_{0}<\eta$. For every $x \in A$ we can easily construct a function $x^{\prime} \in A_{\alpha_{0}}$ such that $x^{\prime} \circ \varphi_{\alpha_{0}}=x$. But this means that $A \subseteq A_{\alpha_{0}} \in \mathcal{J}$ which is a contradiction.

It occurs that we can find also some bounds for non $\left(\mathcal{J}_{\kappa}\right)$ which depend only on $\kappa$. For any infinite cardinal number $\kappa$ we define

$$
\log (\kappa)=\min \left\{\lambda: 2^{\lambda} \geq \kappa\right\}
$$

Theorem 3.3. $\operatorname{non}\left(\mathcal{J}_{\kappa}\right) \geq \log (\kappa)$.

Proof. We show that every subset of $2^{\kappa}$ of cardinality strictly smaller than $\log (\kappa)$ is in $\mathcal{J}_{\kappa}$. Let $\lambda<\log (\kappa)$ and $A=\left\{x_{\alpha}: \alpha<\lambda\right\}$. For every $\xi<\kappa$ we define a function $f_{\xi} \in 2^{\lambda}$ in the following way:

$$
f_{\xi}(\alpha)=x_{\alpha}(\xi)
$$

We have $2^{\lambda}<\kappa$ so we can find a countable infinite set of indices $P \subseteq \kappa$ and a function $f \in 2^{\lambda}$ such that for every $\xi \in P$ we have $f_{\xi}=f$. Straight from the definition of $f_{\xi}$ we obtain that for every $\alpha \in \lambda$ the function $x_{\alpha}\lceil P$ is constant. Thus if $\varphi$ is any member of $\operatorname{Inj}(\omega, \kappa)$ such that $\operatorname{rng}(\varphi)=P$ then the set $\varphi * A$ has at most two elements and, consequently, is in $\mathcal{J}$.

Theorem 3.4. If $2^{\lambda} \geq \kappa$ then $\operatorname{non}\left(\mathcal{J}_{\kappa}\right) \leq \lambda^{\omega}$.

Proof. The proof is based on an idea from [5]. According to the Hewitt-MarczewskiPondiczery Theorem (see [4]) there exists a dense subset $D$ of $2^{\kappa}$ of cardinality $\lambda$. As the space $2^{\kappa}$ is compact so every sequence $s \in D^{\omega}$ has a cluster point $s^{*}$. Let

$$
D^{*}=\left\{s^{*}: s \in D^{\omega}\right\}
$$

We will show that $D^{*} \notin \mathcal{J}_{\kappa}$.

Let $\varphi$ be any member of $\operatorname{Inj}(\omega, \kappa)$. We fix $x \in 2^{\kappa}$ and for each natural number $n$ we choose a function $x_{n} \in D$ such that

$$
(\forall i<n) x_{n}(\varphi(i))=x(\varphi(i))
$$

Let $s^{*}$ be a cluster point of the sequence $s=\left\{x_{n}: n<\omega\right\} \in D^{\omega}$ which is in $D^{*}$. Then

$$
s^{*}\lceil\operatorname{rng}(\varphi)=x\lceil\operatorname{rng}(\varphi) .
$$

But it means that $\varphi * D^{*}=2^{\omega}$ so none $\varphi \in \operatorname{Inj}(\omega, \kappa)$ can be a witness for $D^{*}$.

Corollary 3.5. $\log (\kappa) \leq \operatorname{non}\left(\mathcal{J}_{\kappa}\right) \leq(\log (\kappa))^{\omega}$.

Corollary 3.6. $\operatorname{non}\left(\mathcal{J}_{2^{\kappa}}\right) \leq \kappa^{\omega}$.

A consequence of these results is that the sequence non $\left(\mathcal{J}_{\kappa}\right)$ cannot be constant. However, it is bounded by $\mathfrak{c}$ for long. 
Corollary 3.7. (a) $\operatorname{non}\left(\mathcal{J}_{2^{\mathfrak{c}}}\right) \leq \mathfrak{c}$,

(b) There exists $\kappa=\left(2^{\mathfrak{c}}\right)^{+}$such that $\operatorname{non}\left(\mathcal{J}_{\kappa}\right)>\operatorname{non}(\mathcal{J})$,

(c) If $2^{\mathfrak{c}^{+}}>2^{\mathfrak{c}}$ then for each cardinal number $\xi$ such that $\left(2^{\mathfrak{c}}\right)^{+} \leq \xi \leq 2^{\mathfrak{c}^{+}}$we have $\operatorname{non}\left(\mathcal{J}_{\xi}\right)=\mathfrak{c}^{+}$.

Proof. Applying Corollary 3.6 for $\kappa=\mathfrak{c}$ we obtain (a). Moreover, according to Theorem 3.3 for $\kappa=\left(2^{\mathfrak{c}}\right)^{+}$we have $\operatorname{non}\left(\mathcal{J}_{\kappa}\right) \geq \log (\kappa)>\mathfrak{c}$. But $\operatorname{non}(\mathcal{J}) \leq \mathfrak{c}$ which ends the proof of (b). Finally, Corollary 3.6 for $\kappa=\mathfrak{c}^{+}$together with the previous observation give us the proof of (c).

The following theorem was proved in a weaker version by Fremlin in [6] for the $\sigma$-ideal of null sets.

Theorem 3.8. If $\operatorname{cf}(\kappa)>\omega$ then $\operatorname{non}\left(\mathcal{J}_{\kappa}\right) \leq \max \left\{\operatorname{cf}(\kappa), \sup \left\{\operatorname{non}\left(\mathcal{J}_{\lambda}\right): \lambda<\kappa\right\}\right\}$.

Proof. Let $\left\{\lambda_{\alpha}: \alpha<\operatorname{cf}(\kappa)\right\}$ be a cofinal sequence in $\kappa$. For each $\alpha<\operatorname{cf}(\kappa)$ let $A_{\alpha} \subseteq 2^{\lambda_{\alpha}}$ be a set which is not in $\mathcal{J}_{\lambda_{\alpha}}$ such that $\left|A_{\alpha}\right|=\operatorname{non}\left(\mathcal{J}_{\lambda_{\alpha}}\right)$. For each $f \in A_{\alpha}$ we fix $f^{*} \in 2^{\kappa}$ such that $f^{*}\left\lceil\lambda_{\alpha}=f\right.$. Let $A_{\alpha}^{*}=\left\{f^{*}: f \in A_{\alpha}\right\}$ and let $A^{*}=\bigcup_{\alpha<\operatorname{cf}(\kappa)} A_{\alpha}^{*}$. Obviously,

$$
|A|^{*} \leq \sum_{\alpha<\operatorname{cf}(\kappa)}\left|A_{\alpha}\right| \leq \max \left\{\operatorname{cf}(\kappa), \sup \left\{\operatorname{non}\left(\mathcal{J}_{\lambda}\right): \lambda<\kappa\right\}\right\}
$$

To complete the proof we have to show that $A^{*}$ is not in $\mathcal{J}_{\kappa}$. Suppose otherwise and let $\varphi \in \operatorname{Inj}(\omega, \kappa)$ be a witness for $A^{*}$. Uncountability of $\operatorname{cf}(\kappa)$ implies that $\operatorname{rng}(\varphi) \subseteq \lambda_{\alpha}$ for some $\alpha<\operatorname{cf}(\kappa)$. Thus we can treat $\varphi$ as a member of $\operatorname{Inj}\left(\omega, \lambda_{\alpha}\right)$, too. So

$$
\varphi * A_{\alpha}=\varphi * A_{\alpha}^{*} \subseteq \varphi * A^{*} \in \mathcal{J} .
$$

But $\varphi * A_{\alpha} \notin \mathcal{J}$, which is a contradiction.

The next theorem is a powerful tool which allows us to show several absolute inequalities concerning non's as well as some consistency results.

If $f$ and $g$ are functions with the same domain $D$ then we define

$$
E(f, g)=\{x \in D: f(x)=g(x)\}
$$

Let us observe that the cardinal function $\operatorname{cov}(\mathcal{J})$ can be considered not only for ideals but also for arbitrary families of sets.

Theorem 3.9. Suppose that there exists a family $\mathcal{A} \subseteq \lambda^{\kappa}$ such that $|\mathcal{A}|=\eta$ and

$$
\operatorname{cov}(\{E(f, g): f, g \in \mathcal{A} \& f \neq g\})>\omega .
$$

Then $\operatorname{non}\left(\mathcal{J}_{\eta}\right) \leq \max \left\{\kappa, \operatorname{non}\left(\mathcal{J}_{\lambda}\right)\right\}$.

Proof. Let $\mathcal{A}=\left\{f_{\alpha}: \alpha<\eta\right\}$. To begin with, we show that for every $I \in[\eta]^{\omega}$ there exists $\xi_{I}<\kappa$ such that for every different $\alpha, \beta \in I$ we have $f_{\alpha}\left(\xi_{I}\right) \neq f_{\beta}\left(\xi_{I}\right)$. In order to do this fix $I \in[\eta]^{\omega}$. From the assumption we know that

$$
\bigcap_{\{\alpha, \beta\} \in[I]^{2}} E^{c}\left(f_{\alpha}, f_{\beta}\right) \neq \emptyset
$$


Let $\xi_{I}$ be any member of this intersection. It is easy to check that $\xi_{I}$ fulfils the needed condition.

Now, let $A$ be a subset of $2^{\lambda}$ which is not in $\mathcal{J}_{\lambda}$. For every $x \in A$ and $\xi<\kappa$ we define a function $x_{\xi} \in 2^{\eta}$ in the following way

$$
x_{\xi}(\alpha)=x\left(f_{\alpha}(\xi)\right) \text {. }
$$

Let $A^{*}=\left\{x_{\xi}: x \in A \quad \& \quad \xi<\kappa\right\}$. We will show that $A^{*} \notin \mathcal{J}_{\eta}$.

Suppose otherwise and let $\varphi \in \operatorname{Inj}(\omega, \eta)$ be a witness for $A^{*}$. In particular, the set $\varphi *\left\{x_{\xi_{\operatorname{rng}(\varphi)}}: x \in A\right\}$ is in $\mathcal{J}$. We define a function $\psi$ in the following way

$$
\psi(n)=f_{\varphi(n)}\left(\xi_{\mathrm{rng}(\varphi)}\right)
$$

Straight from the definition of $\xi_{\mathrm{rng}(\varphi)}$ we obtain that $\psi \in \operatorname{Inj}(\omega, \kappa)$. Simple calculations show that $\psi * A=\varphi *\left\{x_{\xi_{\mathrm{rng}(\varphi)}}: x \in A\right\}$, which is a contradiction.

In order to apply this theorem efficiently, we look for a relatively big family $\mathcal{A} \subseteq \kappa^{\kappa}$ such that every pair of functions from this family agree on the set from a certain $\sigma$-ideal of subsets of $\kappa$. The easiest such $\sigma$-ideal is $[\kappa]^{<\kappa}$, on condition $\operatorname{cf}(\kappa)>\omega$.

We say that two functions $f, g \in \lambda^{\kappa}$ are eventually different if there exists $\alpha<\kappa$ such that $E(f, g) \subseteq \alpha$ (in particular, $\left.E(f, g) \in[\kappa]^{<\kappa}\right)$.

Theorem 3.10. If $\operatorname{cf}(\kappa)>\omega$ then $\operatorname{non}\left(\mathcal{J}_{\kappa^{+}}\right) \leq \max \left\{\kappa, \operatorname{non}\left(\mathcal{J}_{\kappa}\right)\right\}$.

Proof. Using the standard diagonal argument we can show that there exists a family $\mathcal{A} \subseteq \kappa^{\kappa}$ of pairwise eventually different functions of size $\kappa^{+}$. Thanks to uncountable cofinality of $\kappa$ the assumption of Theorem 3.9 is fulfilled and we are done.

As a special case of this theorem we obtain a quite surprising result. It was proved by Veličkovič in a special case of meagre sets but the proof remained unpublished

Corollary 3.11. For any productive $\sigma-$ ideal $\mathcal{J}$ we have $\operatorname{non}(\mathcal{J})=\operatorname{non}\left(\mathcal{J}_{\omega_{1}}\right)=$ $\operatorname{non}\left(\mathcal{J}_{\omega_{2}}\right)$.

Proof. According to Fact 3.1 it is enough to $\operatorname{show}$ that $\operatorname{non}(\mathcal{J}) \geq \operatorname{non}\left(\mathcal{J}_{\omega_{1}}\right) \geq$ $\operatorname{non}\left(\mathcal{J}_{\omega_{2}}\right)$. Applying Theorem 3.2 (and Fact 1.6) we obtain

$$
\operatorname{non}\left(\mathcal{J}_{\omega_{1}}\right) \leq \max \left\{c_{\omega_{1}}, \operatorname{non}(\mathcal{J})\right\}=\max \left\{\omega_{1}, \operatorname{non}(\mathcal{J})\right\}=\operatorname{non}(\mathcal{J})
$$

Furthermore, we get from Theorem 3.10 that $\operatorname{non}\left(\mathcal{J}_{\omega_{2}}\right) \leq \max \left\{\omega_{1}, \operatorname{non}\left(\mathcal{J}_{\omega_{1}}\right)\right\}=$ $\operatorname{non}\left(\mathcal{J}_{\omega_{1}}\right)$ which ends the proof.

This result cannot be improved. For instance, assuming Generalized Continuum Hypothesis we obtain

$$
\omega_{1}=\operatorname{non}(\mathcal{J})=\operatorname{non}\left(\mathcal{J}_{\omega_{1}}\right)=\operatorname{non}\left(\mathcal{J}_{\omega_{2}}\right)<\operatorname{non}\left(\mathcal{J}_{\omega_{3}}\right)=\omega_{2}
$$

Next two corollaries are other examples of applying Theorem 3.10 and give us slightly stronger results concerning $\operatorname{non}\left(\mathcal{J}_{\kappa}\right)$ in some special cases. 
Corollary 3.12. If $\kappa<\omega_{\omega}$ then $\operatorname{non}\left(\mathcal{J}_{\kappa^{+}}\right) \leq \max \{\kappa, \operatorname{non}(\mathcal{J})\}$.

Proof. It is enough to iterate Theorem 3.10 finitely many times and use Corollary 3.11 .

Corollary 3.13. If $\operatorname{non}(\mathcal{J})=\omega_{n}$ for some natural number $n$ then $\operatorname{non}(\mathcal{J})=$ $\operatorname{non}\left(\mathcal{J}_{\omega_{n+1}}\right)$.

Proof. We obtain from Corollary 3.12 that $\operatorname{non}\left(\mathcal{J}_{\omega_{n+1}}\right) \leq \max \left\{\omega_{n}, \operatorname{non}(\mathcal{J})\right\}=\omega_{n}$. Hence, according to Fact 3.1 the proof is finished.

In [9] we can find another example of a big family of pairwise disjoint functions which allows us to formulate the following theorem.

Theorem 3.14. $\operatorname{non}\left(\mathcal{J}_{2^{\kappa}+}\right) \leq \max \left\{\kappa^{+}, \operatorname{non}\left(\mathcal{J}_{2^{\kappa}}\right)\right\}$.

Proof. For every $X \subseteq \kappa^{+}$we define a function $f_{X}: \kappa^{+} \rightarrow\left[\kappa^{+}\right] \leq \kappa$ (and $\left|\left[\kappa^{+}\right] \leq \kappa\right|=$ $\left.2^{\kappa}\right)$ in the following way:

$$
f_{X}(\alpha)=X \cap \alpha
$$

Let $\mathcal{A}=\left\{f_{X}: X \subseteq \kappa^{+}\right\}$. Thus $|\mathcal{A}|=2^{\kappa^{+}}$. Obviously, for different $X, Y \subseteq \kappa^{+}$we have $E\left(f_{X}, f_{Y}\right) \subseteq \alpha<\kappa^{+}$for some $\alpha$ and thanks to the regularity of $\kappa^{+}$we can apply Theorem 3.9, which ends the proof.

As before, we can extract from this theorem some interesting corollaries.

Corollary 3.15. If $\xi$ is a cardinal number such that $\mathfrak{c} \leq \xi \leq 2^{\omega_{1}}$ then $\operatorname{non}\left(\mathcal{J}_{\xi}\right)=$ $\operatorname{non}\left(\mathcal{J}_{\mathfrak{c}}\right)$.

Proof. It is enough to apply Theorem 3.14 for $\kappa=\omega$.

Corollary 3.16. If $\kappa<\omega_{\omega}$ then $\operatorname{non}\left(\mathcal{J}_{2^{\kappa}}\right) \leq \max \left\{\kappa, \operatorname{non}\left(\mathcal{J}_{\mathfrak{c}}\right)\right\}$.

Proof. It is enough to iterate Theorem 3.14 finitely many times and use Corollary 3.15 .

In general, constructing big families of pairwise eventually different functions demands sophisticated combinatorial assumptions. For example, we have the following fact.

Fact 3.17. ([10], Theorem 3.1) Assume that $\mathfrak{c}<\omega_{\omega_{1}}$ and $\mathfrak{c}<2^{\omega_{1}}$. Then there exists a family $\mathcal{A} \subseteq \omega_{1}^{\omega_{1}}$ of pairwise eventually different functions of size $2^{\omega_{1}}$.

Corollary 3.18. Under the assumptions of Fact 3.17, if $\xi \leq 2^{\omega_{1}}$ is an infinite cardinal number then $\operatorname{non}\left(\mathcal{J}_{\xi}\right)=\operatorname{non}(\mathcal{J})$.

Proof. Straightforward from Theorem 3.9 and Fact 3.17.

Question. Is it consistent with $\mathrm{ZFC}$ that $\operatorname{non}(\mathcal{J})<\operatorname{non}\left(\mathcal{J}_{\mathfrak{c}}\right)$ ?

\section{Covering of $\mathcal{J}_{\kappa}$}

As in a case of the uniformity, no straightforward formula for the covering of $\mathcal{J}_{\kappa}$ was found. However, the situation is different because $\operatorname{cov}\left(\mathcal{J}_{\kappa}\right)$ form a decreasing sequence. 
Fact 4.1. If $\lambda \leq \kappa$ then $\operatorname{cov}\left(\mathcal{J}_{\lambda}\right) \geq \operatorname{cov}\left(\mathcal{J}_{\kappa}\right)$.

Proof. Let $\left\{A_{\xi}: \xi<\eta\right\} \subseteq \mathcal{J}_{\lambda}$ be a covering family for $2^{\lambda}$ (i.e. $\bigcup_{\xi<\eta} A_{\xi}=2^{\lambda}$ ). We notice that if $A \subseteq \mathcal{J}_{\lambda}$ with a witness $\varphi \in \operatorname{Inj}(\omega, \lambda)$ then a set

$$
K_{A}=\left\{x \in 2^{\kappa}: x\lceil\lambda \in A\}\right.
$$

is in $\mathcal{J}_{\kappa}$ with the same witness, treated as a member of $\operatorname{Inj}(\omega, \kappa)$.

To finish the proof we observe that a family $\left\{K_{A_{\xi}}: \xi<\eta\right\} \subseteq \mathcal{J}_{\kappa}$ is a covering family for $2^{\kappa}$.

This fact implies that results concerning coverings of $\mathcal{J}_{\kappa}$ 's are of totally different type than theorems proved so far.

Theorem 4.2. If $\operatorname{cov}\left(\mathcal{J}_{\kappa}\right) \leq \lambda \leq \kappa$ then $\operatorname{cov}\left(\mathcal{J}_{\kappa}\right)=\operatorname{cov}\left(\mathcal{J}_{\lambda}\right)$.

Proof. According to Fact 4.1 it is enough to show that $\operatorname{cov}\left(\mathcal{J}_{\kappa}\right) \geq \operatorname{cov}\left(\mathcal{J}_{\lambda}\right)$.

Let $\left\{A_{\xi}: \xi<\eta\right\} \subseteq \mathcal{J}_{\kappa}$ be a covering family for $2^{\kappa}$ and for each $\xi<\eta$ let $\varphi_{\xi} \in$ $\operatorname{Inj}(\omega, \kappa)$ be a witness for $A_{\xi}$. We know that $\eta \leq \lambda$ so there exists a set $B \subseteq \kappa$ such that

$$
\bigcup_{\xi<\eta} \operatorname{rng}\left(\varphi_{\xi}\right) \subseteq B
$$

and we can find a bijection $\Phi$ from $\lambda$ onto $B$. Thus for each $\xi<\eta$ the set $\Phi * A_{\xi}=$ $\left\{x \circ \Phi: x \in A_{\xi}\right\} \subseteq 2^{\lambda}$ is in $\mathcal{J}_{\lambda}$ (because the function $\Phi^{-1} \circ \varphi_{\xi} \in \operatorname{Inj}(\omega, \lambda)$ is its witness).

Fix $x \in 2^{\lambda}$. Let $x^{\prime}$ be any function from $2^{\kappa}$ such that $x^{\prime} \uparrow B=x \circ \Phi^{-1} \uparrow B$. Then $x^{\prime} \in A_{\xi_{0}}$ for some $\xi_{0}<\eta$ and $x=x^{\prime} \circ \Phi \in \Phi * A_{\xi_{0}}$. Hence the family $\left\{\Phi * A_{\xi}: \xi<\eta\right\}$ is a covering family for $2^{\lambda}$.

Let us consider a function from infinite cardinal numbers into themselves given by a formula $F(\kappa)=\operatorname{cov}\left(\mathcal{J}_{\kappa}\right)$. According to Fact 4.1 this function is decreasing. The following corollaries show that its diagram intersects nonempty with the diagonal and the function stabilizes from this point.

Corollary 4.3. If $\kappa \geq \mathfrak{c}$ then $\operatorname{cov}\left(\mathcal{J}_{\kappa}\right)=\operatorname{cov}\left(\mathcal{J}_{\mathfrak{c}}\right)$.

Proof. (cf [5], Theorem 6.17 (d)(iii)) Notice that $\operatorname{cov}(\mathcal{J}) \leq \mathfrak{c}$ as the family of all singletons is a covering family for $2^{\omega}$. Therefore, we have $\operatorname{cov}\left(\mathcal{J}_{\kappa}\right) \leq \operatorname{cov}(\mathcal{J}) \leq \mathfrak{c} \leq \kappa$ and it is enough to apply Theorem 4.2.

Corollary 4.4. If $\operatorname{cov}\left(\mathcal{J}_{\mathfrak{c}}\right)=\lambda$ then $\operatorname{cov}\left(\mathcal{J}_{\lambda}\right)=\lambda$.

Proof. We know from Fact 4.1 that $\operatorname{cov}\left(\mathcal{J}_{\kappa}\right) \leq \operatorname{cov}(\mathcal{J}) \leq \mathfrak{c}$. Applying Theorem 4.2 to $\kappa=\mathfrak{c}$ we obtain that $\operatorname{cov}\left(\mathcal{J}_{\lambda}\right)=\operatorname{cov}\left(\mathcal{J}_{\mathfrak{c}}\right)=\lambda$.

Corollary 4.5. If $\operatorname{cov}\left(\mathcal{J}_{\lambda}\right)>\lambda$ for some cardinal number $\lambda$ then $(\forall \kappa \geq \lambda) \operatorname{cov}\left(\mathcal{J}_{\kappa}\right)>$ $\lambda$.

Proof. It is enough to show that if there exists a cardinal number $\kappa$ such that $\kappa \geq \lambda$ and $\operatorname{cov}\left(\mathcal{J}_{\kappa}\right) \leq \lambda$ then $\operatorname{cov}\left(\mathcal{J}_{\lambda}\right) \leq \lambda$. But this is true according to Theorem 4.2.

If the covering of $\mathcal{J}_{\kappa}$ is relatively small we have a bound for it depending only on $\kappa$. 
Theorem 4.6. If $\operatorname{cov}\left(\mathcal{J}_{\kappa}\right)<\operatorname{add}(\mathcal{J})$ then $\operatorname{cov}\left(\mathcal{J}_{\kappa}\right) \leq c_{\kappa}$.

Proof. Let $\left\{A_{\xi}: \xi<\eta\right\} \subseteq \mathcal{J}_{\kappa}$ be a covering family for $2^{\kappa}$ and for each $\xi<\eta$ let $\psi_{\xi} \in \operatorname{Inj}(\omega, \kappa)$ be a witness for $A_{\xi}$. Let $\left\{\varphi_{\alpha}: \alpha<c_{\kappa}\right\} \subseteq \operatorname{Inj}(\omega, \kappa)$ be a family such that $\left\{\operatorname{rng}\left(\varphi_{\alpha}\right): \alpha<c_{\kappa}\right\}$ is a base of the $\sigma$-ideal of all countable subsets of $\kappa$. We have to show that if $\eta<\operatorname{add}(\mathcal{J})$ then we can construct a covering family for $2^{\kappa}$ of cardinality at most $c_{\kappa}$.

For each $\alpha<c_{\kappa}$ we define a set

$$
T_{\alpha}=\left\{\xi<\eta: \operatorname{rng}\left(\psi_{\xi}\right) \subseteq \operatorname{rng}\left(\varphi_{\alpha}\right)\right\}
$$

According to the Lemma 1.5 the function $\varphi_{\alpha}$ is a common witness for all $A_{\xi}$ such that $\xi \in T_{\alpha}$. But the set $T_{\alpha}$ has cardinality strictly smaller than $\operatorname{add}(\mathcal{J})$, which means that

$$
\varphi_{\alpha} *\left(\bigcup_{\xi \in T_{\alpha}} A_{\xi}\right)=\bigcup_{\xi \in T_{\alpha}}\left(\varphi_{\alpha} * A_{\xi}\right) \in \mathcal{J} .
$$

Let $C_{\alpha}=\bigcup_{\xi \in T_{\alpha}} A_{\xi}$. We showed above that $C_{\alpha} \in \mathcal{J}_{\kappa}$. Moreover, we know that $\bigcup_{\alpha<c_{\kappa}} C_{\alpha}=\bigcup_{\xi<\eta} A_{\xi}$ (because $\bigcup_{\alpha<c_{\kappa}} T_{\alpha}=\eta$ ) so the family $\left\{C_{\alpha}: \alpha<c_{\kappa}\right\}$ is a covering family for $2^{\kappa}$.

Corollary 4.7. If $\operatorname{cov}\left(\mathcal{J}_{\omega_{1}}\right)<\operatorname{add}(\mathcal{J})$ then $\left(\forall \kappa \geq \omega_{1}\right) \operatorname{cov}\left(\mathcal{J}_{\kappa}\right)=\omega_{1}$.

Proof. We deduce from Fact 1.6 that $c_{\omega_{1}}=\omega_{1}$ and then use Fact 4.1 and Theorem 4.6.

If we add certain assumptions about $\mathcal{J}$ and $\mathfrak{c}$, we will be able to show that the range of a function $F(x)=\operatorname{cov}\left(\mathcal{J}_{x}\right)$ has at most two elements. If the function $F$ is not constant then we can precisely show the place where it changes its value.

Corollary 4.8. If $\operatorname{add}(\mathcal{J})=\operatorname{cov}(\mathcal{J})$ then for every natural number $n$

$$
\operatorname{cov}\left(\mathcal{J}_{\omega_{n}}\right)<\operatorname{cov}(\mathcal{J}) \Rightarrow \operatorname{cov}\left(\mathcal{J}_{\omega_{n}}\right) \leq \omega_{n}
$$

Proof. As in the previous proof using the fact that for every natural number $n$ we have $c_{\omega_{n}}=\omega_{n}$.

Corollary 4.9. If $\operatorname{add}(\mathcal{J})=\operatorname{cov}(\mathcal{J})$ and $\mathfrak{c}=\omega_{n}$ where $n$ is a natural number then there exists at most one natural number $m$ such that $\operatorname{cov}\left(\mathcal{J}_{\omega_{m}}\right)>\operatorname{cov}\left(\mathcal{J}_{\omega_{m+1}}\right)$. If such $m$ really exists then $\operatorname{cov}\left(\mathcal{J}_{\omega_{m+1}}\right)=\operatorname{cov}\left(\mathcal{J}_{\mathfrak{c}}\right)=\omega_{m+1}$.

Proof. Let $n$ be a natural number. We know from Corollary 4.4 that if $\operatorname{cov}\left(\mathcal{J}_{\mathfrak{c}}\right)=$ $\omega_{m+1}$ for some $m \in \omega$ then $\operatorname{cov}\left(\mathcal{J}_{\kappa}\right)=\omega_{m+1}$ for all $\kappa$ such that $\omega_{m+1} \leq \kappa \leq \mathfrak{c}$.

On the other hand if $\operatorname{cov}\left(\mathcal{J}_{\omega_{i}}\right)>\operatorname{cov}\left(\mathcal{J}_{\omega_{i+1}}\right)$ for some $i<m$ then $\operatorname{cov}(\mathcal{J})>$ $\operatorname{cov}\left(\mathcal{J}_{\omega_{i+1}}\right)$ and we know from Corollary 4.8 that

$$
\operatorname{cov}\left(\mathcal{J}_{\omega_{i+1}}\right) \leq \omega_{i+1}<\omega_{m+1}=\operatorname{cov}\left(\mathcal{J}_{\omega_{m+1}}\right)
$$

which contradicts Fact 4.1. 


\section{Several MOdels}

In this section we investigate the cardinal coefficients of classical $\sigma$-ideals in some models of the Set Theory. Let us assume, as usual, that $\mathcal{J}$ is a proper and productive $\sigma$-ideal of subsets of $2^{\omega}$ containing all points.

Theorem 5.1. Let $\kappa$ be an uncountable cardinal number. If GCH holds then

(a) $\operatorname{add}\left(\mathcal{J}_{\kappa}\right)=\operatorname{cov}\left(\mathcal{J}_{\kappa}\right)=\omega_{1}$.

(b) (1) If $\operatorname{cf}(\kappa)=\omega$ then $\operatorname{non}\left(\mathcal{J}_{\kappa}\right)=\kappa^{+}$;

(2) if $\kappa=\lambda^{+}$and $\operatorname{cf}(\lambda)>\omega$ then $\operatorname{non}\left(\mathcal{J}_{\kappa}\right)=\lambda$;

(3) otherwise, $\operatorname{non}\left(\mathcal{J}_{\kappa}\right)=\kappa$.

(c) If $\kappa \geq \omega_{2}$ then

(1) If $\operatorname{cf}(\kappa)=\omega$ then $\operatorname{cof}\left(\mathcal{J}_{\kappa}\right)=\kappa^{+}$;

(2) If $\operatorname{cf}(\kappa)>\omega$ then $\operatorname{cof}\left(\mathcal{J}_{\kappa}\right)=\kappa$.

Proof. (cf [5], Theorem 6.18)

(a) Obvious from Fact 4.1 .

(b) If $\kappa=\lambda^{+}$then $\log (\kappa)=\lambda$ and according to the Corollary 3.5 we have $\lambda \leq$ $\operatorname{non}\left(\mathcal{J}_{\kappa}\right) \leq \lambda^{\omega} \leq \lambda^{+}$. Thus if $\operatorname{cf}(\lambda)>\omega$ then $\lambda^{\omega}=\lambda$, so $\operatorname{non}\left(\mathcal{J}_{\kappa}\right)=\lambda$. If $\operatorname{cf}(\lambda)=\omega$ then $\operatorname{non}\left(\mathcal{J}_{\kappa}\right)$ must be $\lambda^{+}$because it has an uncountable cofinality.

If $\kappa$ is a limit cardinal number then $\log (\kappa)=\kappa$ so $\kappa \leq \operatorname{non}\left(\mathcal{J}_{\kappa}\right) \leq \kappa^{\omega} \leq \kappa^{+}$by Corollary 3.5. As before, $\operatorname{cf}(\kappa)>\omega$ implies that $\operatorname{non}\left(\mathcal{J}_{\kappa}\right)=\kappa$ and $\operatorname{cf}(\kappa)=\omega$ implies that $\operatorname{non}\left(\mathcal{J}_{\kappa}\right)=\kappa^{+}$.

(c) As $\operatorname{cof}(\mathcal{J}) \leq 2^{\mathfrak{c}}=\omega_{2}$ so we deduce from Theorems 2.2 and 2.3 and Fact 1.6 that for $\kappa \geq \omega_{2}$ we have

$$
\operatorname{cof}\left(\mathcal{J}_{\kappa}\right)=c_{\kappa}=\kappa^{\omega}
$$

As in the previous part of this proof, $\operatorname{cf}(\kappa)>\omega \operatorname{implies} \operatorname{cof}\left(\mathcal{J}_{\kappa}\right)=\kappa$ and $\operatorname{cf}(\kappa)=\omega$ implies $\operatorname{cof}\left(\mathcal{J}_{\kappa}\right)=\kappa^{+}$(because $\operatorname{cf}\left(\operatorname{cof}\left(\mathcal{J}_{\kappa}\right)\right)$ is uncountable).

Remark. We are not able to compute precisely $\operatorname{cof}\left(\mathcal{J}_{\omega_{1}}\right)$ in the previous theorem without any extra assumptions. But for example, if $\operatorname{cof}(\mathcal{J})=\mathfrak{c}=\omega_{1}$ (for instance, $\mathcal{J}$ has a base consisting of Borel sets) then $\operatorname{cof}\left(\mathcal{J}_{\omega_{1}}\right)=\omega_{1}$. Moreover, if $\mathcal{J}$ has WFP then $\operatorname{cof}\left(\mathcal{J}_{\omega_{1}}\right)=\operatorname{cof}(\mathcal{J})$.

Another interesting result can be obtained by applying Martin's Axiom (MA).

Theorem 5.2. If $M A$ holds then for each $\kappa \leq 2^{\mathfrak{c}}$ we have $\operatorname{non}\left(\mathcal{J}_{\kappa}\right)=\operatorname{non}(\mathcal{J})=\mathfrak{c}$.

Proof. Straight from definitions we have $\mathfrak{s} \leq \aleph_{0}-\mathfrak{s}$, where $\mathfrak{s}$ is the classical splitting number. We know that under MA we have $\mathfrak{s}=\mathfrak{c}$ (see for example [16]). Therefore $\operatorname{non}\left(\mathcal{S}_{2}\right)=\aleph_{0}-\mathfrak{s}=\mathfrak{c}$. As it is easy to observe, if $\mathcal{I} \subseteq \mathcal{J}$ then $\operatorname{non}(\mathcal{I}) \leq \operatorname{non}(\mathcal{J})$. Thus $\operatorname{non}(\mathcal{J})=\mathfrak{c}$. So it is enough to apply Fact 3.1 and the Corollary 3.7.

Remark. As one can see, we can obtain the same result replacing MA by a weaker combinatorial property i.e. $\aleph_{0}-\mathfrak{s}=\mathfrak{c}$. It is worth observing that assuming another property (which is also implied by MA), namely $(\forall \omega \leq \kappa<\mathfrak{c})\left(2^{\kappa}=\mathfrak{c}\right.$ ) we get another, weaker result: $\operatorname{non}\left(\mathcal{J}_{\mathfrak{c}^{+}}\right)=\mathfrak{c}$. The proof is straightforward from Corollary 3.5 .

The above results are true for every productive $\sigma$-ideal $\mathcal{J}$. Now we focus on the $\sigma$-ideal of meagre sets. 
The set $A$ is called a $(\kappa, \lambda)-$ Luzin set for a $\sigma$-ideal $\mathcal{J}$ if $A$ is of cardinality $\kappa$ and for every set $B \in \mathcal{J}$ we have $|A \cap B|<\lambda$. For more information about Luzin sets see [2]. In particular, the following fact holds.

Fact 5.3. Let $\kappa$ be an uncountable cardinal number. If there exists a $\left(\kappa, \omega_{1}\right)-$ Luzin set for a $\sigma$-ideal $\mathcal{J}$ then $\operatorname{cov}(\mathcal{J}) \geq \kappa$ and $\operatorname{non}(\mathcal{J})=\omega_{1}$.

In our further considerations we will use the standard method of forcing (see [8] for more details) to obtain required models.

Theorem 5.4. Let $\lambda$ be a cardinal number of uncountable cofinality and assume that $G C H$ holds in the ground model $\mathbf{V}$. Let $\mathbf{V}[\mathrm{G}]$ be a generic extension obtained by adding $\lambda$ Cohen reals to the ground model. Then in $\mathbf{V}[\mathrm{G}]$

(a) $(\forall \kappa \geq \omega)\left(\operatorname{cov}\left(\mathcal{M}_{\kappa}\right)=\lambda=\mathfrak{c}\right)$;

(b) $(\forall \omega \leq \kappa \leq \lambda)\left(\operatorname{non}\left(\mathcal{M}_{\kappa}\right)=\omega_{1}\right)$;

(c) for $\kappa \geq \lambda$, non $\left(\mathcal{M}_{\kappa}\right)$ is computed as in the Theorem 5.1.

Proof. It is well-known (see for example [14]) that $\mathbf{V}[\mathrm{G}] \models \mathfrak{c}=\lambda$ and that added Cohen reals form a $\left(\lambda, \omega_{1}\right)$-Luzin set for $\mathcal{M}$. Moreover, for each $\kappa \leq \lambda$ there exists also a $\left(\lambda, \omega_{1}\right)$-Luzin set for $\mathcal{M}_{\kappa}$. It can be easily obtained by glueing the Cohen reals into functions from $2^{\kappa}$. Then straight from Fact 5.3 and Corollary 4.3 we get (a) and (b). To prove (c) it is enough to observe that $\mathbf{V}[\mathrm{G}] \models 2^{\kappa}=\kappa^{+}$for such $\kappa$ 's. Consequently, we can compute non $\left(\mathcal{M}_{\kappa}\right)$ exactly as in the proof of Theorem 5.1(b) (using Corollary 3.5).

The situations of adding $\kappa$ Cohen reals for $\kappa$ 's of countable cofinality is a bit more complicated.

Theorem 5.5. Assume that GCH holds in the ground model and let $\mathbf{V}[\mathrm{G}]$ be a generic extension obtained by adding $\omega_{\omega}$ Cohen reals to the ground model. Then in $\mathbf{V}[\mathrm{G}]$

(a) $\operatorname{cov}(\mathcal{M})=\omega_{\omega+1}=\mathfrak{c}$ and $\left(\forall \kappa \geq \omega_{1}\right)\left(\operatorname{cov}\left(\mathcal{M}_{\kappa}\right)=\omega_{\omega}\right)$;

(b) $\left(\forall \omega \leq \kappa \leq \omega_{\omega}\right)\left(\operatorname{non}\left(\mathcal{M}_{\kappa}\right)=\omega_{1}\right)$;

(c) for $\kappa \geq \omega_{\omega+2}$, non $\left(\mathcal{M}_{\kappa}\right)$ is computed as in the Theorem 5.1.

Proof. We know that $\mathbf{V}[\mathrm{G}] \models \mathfrak{c}=\omega_{\omega+1}$. The same argument as in the previous proof shows that for each infinite $\kappa \leq \omega_{\omega}$, there exist an $\left(\omega_{\omega}, \omega_{1}\right)$-Luzin set for $\mathcal{M}_{\kappa}$. This proves (b) and shows that for every such $\kappa, \operatorname{cov}\left(\mathcal{M}_{\kappa}\right) \geq \omega_{\omega}$. Miller showed in [15] that $\operatorname{cf}(\operatorname{cov}(\mathcal{M}))$ is uncountable, so $\operatorname{cov}(\mathcal{M})=\omega_{\omega+1}$. In the same paper he proved that $\operatorname{cov}\left(\mathcal{M}_{\omega+1}\right)=\omega_{\omega}$ which together with Corollaries 4.3 and 4.4 end the proof of (a).

Finally, (c) is true as $\mathbf{V}[\mathrm{G}] \models\left(\forall \kappa \geq \omega_{\omega+1}\right)\left(2^{\kappa}=\kappa^{+}\right)$so we can apply Corollary 3.5 to compute $\operatorname{non}\left(\mathcal{M}_{\kappa}\right)$ for $\kappa \geq \omega_{\omega+2}$.

Question. What is the value of $\operatorname{non}\left(\mathcal{M}_{\omega_{\omega+1}}\right)$ in the model described in Theorem $5.5 ?$

Remark. Let us notice that Theorems 5.4 and 5.5 remain true if we replace meagre sets by null sets and Cohen reals by random reals. In the case of the first theorem the proof is analogous. In the case of the latter one we have to use additionally Theorem 3 from [15]. 
We introduce a well-known combinatorial principle called "stick" and denoted by $(\uparrow)$ and a cardinal number $\uparrow$ connected with it. For more details see [7].

Definition. $(\boldsymbol{\uparrow})$ : There exists a family $\mathcal{T} \subseteq\left[\omega_{1}\right]^{\omega}$ of cardinality $\omega_{1}$ such that for any $X \in\left[\omega_{1}\right]^{\omega_{1}}$ there exists $T \in \mathcal{T}$ with $T \subseteq X$.

$\uparrow=\min \left\{|\mathcal{T}|: \mathcal{T} \subseteq\left[\omega_{1}\right]^{\omega} \quad \& \quad\left(\forall X \in\left[\omega_{1}\right]^{\omega_{1}}\right)(\exists T \in \mathcal{T}) T \subseteq X\right\}$

Let us observe that $(\boldsymbol{\uparrow}) \Longleftrightarrow \boldsymbol{\uparrow}=\omega_{1}$. The following simple fact holds.

Fact 5.6. Let $\mathcal{J}$ be any productive and proper $\sigma$-ideal of subsets of $2^{\omega}$ containing all points. Then

$$
\operatorname{cov}\left(\mathcal{J}_{\omega_{1}}\right) \leq \uparrow
$$

Proof. Let $\mathcal{T}$ be any family of countable infinite subsets of $\omega_{1}$ such that for each $X \in\left[\omega_{1}\right]^{\omega_{1}}$ there exists $T \in \mathcal{T}$ with $T \subseteq X$. Let $\mathbf{0}_{T}$ and $\mathbf{1}_{T}$ denote functions constantly equal to 0 and 1 , respectively, on its domain, which is the set $T$. Let $\left[\mathbf{0}_{T}\right]=\left\{x \in 2^{\omega}: x\lceil T \equiv 0\}\right.$ and $\left[\mathbf{1}_{T}\right]=\left\{x \in 2^{\omega}: x\lceil T \equiv 1\}\right.$. Then for every $T \in \mathcal{T}$ both sets $\left[\mathbf{0}_{T}\right]$ and $\left[\mathbf{1}_{T}\right]$ are in $\mathcal{J}$ and any $\varphi \in \operatorname{Inj}\left(\omega, \omega_{1}\right)$ such that $\operatorname{rng}(\varphi)=T$ is their witness. Thus the family

$$
\mathcal{F}=\left\{\left[\mathbf{0}_{T}\right],\left[\mathbf{1}_{T}\right]: T \in \mathcal{T}\right\} \subseteq \mathcal{J}
$$

is a covering family. To prove this it is enough to observe that for any function $x \in 2^{\omega}$ either its pre-image of 0 or its pre-image of 1 is uncountable. Hence we can find a set $T \in \mathcal{T}$ contained in one of these pre-images and, consequently, either $x \in\left[\mathbf{0}_{T}\right]$ or $x \in\left[\mathbf{1}_{T}\right]$.

Now we construct another model in which the function $F(\kappa)=\operatorname{cov}\left(\mathcal{M}_{\kappa}\right)$ falls only once but rapidly.

Theorem 5.7. Let $\kappa$ be an uncountable cardinal number and assume that $G C H$ holds in the ground model. We define a forcing notion $\mathbb{P}_{\kappa}=\{p: p$ is a function \& $\left.\operatorname{dom}(p) \in[\kappa] \leq \omega \quad \& \operatorname{rng}(p) \subseteq \omega^{<\omega}\right\}$. For $p, q \in \mathbb{P}_{\kappa}$ we define

$$
p \leq q \Longleftrightarrow \operatorname{dom}(p) \supseteq \operatorname{dom}(q) \quad \& \quad(\forall \alpha \in \operatorname{dom}(q))(p(\alpha) \supseteq q(\alpha)) .
$$

If $\mathrm{G}$ is a $\mathbb{P}_{\kappa}$-generic filter over $\mathbf{V}$ then $\mathbf{V}[\mathrm{G}] \models \operatorname{cov}(\mathcal{M})=\mathfrak{c} \& \operatorname{cov}\left(\mathcal{M}_{\omega_{1}}\right)=\omega_{1}$.

Proof. This notion of forcing was introduced in a special case $\kappa=\omega_{3}$ by Miller in [15].

As GCH holds in $\mathbf{V}$ then a special case of the $\Delta$-lemma is true in $\mathbf{V}$, namely every family of $\omega_{2}$ countable sets contains a $\Delta$-system of cardinality $\omega_{2}$ (see [12] for more details). This fact implies that $\mathbb{P}_{\kappa}$ has the $\omega_{2}$-chain condition so cardinal numbers bigger then $\omega_{1}$ are preserved, while $\omega_{1}^{\mathbf{V}}$ is collapsed, which was all shown in $[15]$. Hence $\mathbf{V}[\mathrm{G}] \models\left|\omega_{k+1}^{\mathbf{V}}\right|=\omega_{k}$ for every natural number $k$ and $\mathbf{V}[\mathrm{G}] \models\left|\omega_{\xi}^{\mathbf{V}}\right|=\omega_{\xi}$ for $\xi \geq \omega$.

Let us take $g=\bigcup G: \kappa \rightarrow \omega^{\omega}$. One can show using a typical argument from the forcing theory that $g(\alpha)$ is a Cohen real for each ordinal number $\alpha<\kappa$ (in a sense that $g(\alpha)$ does not belong to any meager subset of $\omega^{\omega}$ coded in $\left.\mathbf{V}\right)$. Thus $\mathbb{P}_{\kappa}$ adds $|\kappa|$ many Cohen reals, which form (in $\mathbf{V}[\mathrm{G}])$ a $\left(|\kappa|, \omega_{2}^{\mathbf{V}}\right)$-Luzin set for $\mathcal{M}$ (because $\mathbb{P}_{\kappa}$ satisfies the $\omega_{2}$-chain condition). 
It is well-known that in $\mathbf{V}[\mathrm{G}]$ we have $\mathfrak{c}=|\kappa|$ if $\operatorname{cf}(\kappa)>\omega$ and $\mathfrak{c}=|\kappa|^{+}$otherwise. Similarly as in the proofs of Theorems 5.4 and 5.5 the existence of the $\left(|\kappa|, \omega_{1}\right)$-Luzin set for $\mathcal{M}$ (our Luzin set is a subset of $\omega^{\omega}$ instead of $2^{\omega}$, but cardinal coefficients of $\mathcal{M}$ are the same for any uncountable Polish space without isolated points) implies that $\mathbf{V}[\mathrm{G}] \models \operatorname{cov}(\mathcal{M})=\mathfrak{c}$. To complete the proof we have to show that $\mathbf{V}[\mathrm{G}] \models$ $\operatorname{cov}\left(\mathcal{M}_{\omega_{1}}\right)=\omega_{1}$. In order to do this we need the following claim:

Claim. $\mathbf{V}[\mathrm{G}] \models(\uparrow)$.

Let $\mathbf{V}[\mathrm{G}] \models f: \omega_{1} \rightarrow \omega_{1} \& f$ is an injection and take $p_{0} \in G \cap \mathbb{P}_{\kappa}$ such that $p_{0} \Vdash " \dot{f}: \omega_{2}^{\mathbf{V}} \rightarrow \omega_{2}^{\mathbf{V}} \& \dot{f}$ is an injection". In $\mathbf{V}$ we define a set

$$
D_{f}=\left\{q \in \mathbb{P}_{\kappa}: q \leq p_{0} \&\left(\exists Z \in\left[\omega_{2}\right]^{\omega}\right) q \| \vdash^{*} Z \subseteq \operatorname{rng}(\dot{f})^{\prime}\right\} .
$$

We show that $D_{f}$ is dense in $\mathbb{P}_{\kappa}$ under $p_{0}$.

Fix any $p \leq p_{0}$. For every $\alpha \in \omega_{2}$ there exists $q_{\alpha} \leq p$ and $\beta_{\alpha} \in \omega_{2}$ such that $q_{\alpha} \Vdash " \dot{f}(\alpha)=\beta_{\alpha}$ ". Using the same version of the $\Delta$-lemma as above we get a set $Y \in\left[\omega_{2}\right]^{\omega_{2}}$ such that functions $\left\{q_{\alpha}: \alpha \in Y\right\}$ form a $\Delta$-system (i.e. their domains form a $\Delta$-system and they are equal on its root). Then for any set $T \in[Y]^{\omega}$ we have

$$
q_{T}=\bigcup_{\alpha \in T} q_{\alpha} \in \mathbb{P}_{\kappa}
$$

This implies that for each $\alpha \in T$ we have $q_{T} \Vdash$ " $\dot{f}(\alpha)=\beta_{\alpha}$ ". Thus

$$
q_{T} \Vdash "\left\{\beta_{\alpha}: \alpha \in T\right\} \subseteq \operatorname{rng}(\dot{f}) " .
$$

So $q=q_{T}$ and $Z=\left\{\beta_{\alpha}: \alpha \in T\right\}$. The set $Z$ is infinite as the function $f$ is an injection. Hence $q \leq p_{0}$ and $q \in D_{f}$.

As $D_{f}$ is dense in $\mathbb{P}_{\kappa}$ under $p_{0}$ then there exists $p^{\prime} \leq p_{0}$ such that $p^{\prime} \in G \cap D_{f}$. Then there exists $Z \in\left[\omega_{2}^{\mathbf{V}}\right]^{\omega}$ such that $p^{\prime} \Vdash$ " $Z \subseteq \operatorname{rng}(\dot{f})$ " and, consequently,

$$
\mathbf{V}[\mathrm{G}] \models\left(\exists Z \in\left[\omega_{1}\right]^{\omega} \cap \mathbf{V}\right) Z \subseteq \operatorname{rng}(f) .
$$

To finish the proof of the claim it is enough to observe that if $\mathbf{V}[\mathrm{G}] \models B \in\left[\omega_{1}\right]^{\omega_{1}}$ then there exists in $\mathbf{V}[\mathrm{G}]$ an injection $f: \omega_{1} \rightarrow B \subseteq \omega_{1}$. Thus there exists a set $Z \in\left[\omega_{1}\right]^{\omega} \cap \mathbf{V}$ such that $Z \subseteq \operatorname{rng}(f) \subseteq B$. Hence

$$
\mathbf{V}[\mathrm{G}] \models \boldsymbol{\varphi} \leq\left|\left[\omega_{1}\right]^{\omega} \cap \mathbf{V}\right|
$$

But the cardinality of the family $\left[\omega_{1}\right]^{\omega} \cap \mathbf{V}$ is equal to the cardinality of the family $\left[\omega_{2}^{\mathbf{V}}\right]^{\omega}$ counted in the ground model, which is equal to $\omega_{2}^{\mathbf{V}}$ (as $\mathbf{V} \models$ GCH).

To finish the proof of the theorem it is enough to use Fact 5.6.

Remark. Miller in his paper [15] proved a slightly weaker property of the forcing $\mathbb{P}_{\omega_{3}}$. We proved that in the generic extension obtained by this forcing the principle $\left(\varphi^{\circ}\right)$ holds.

As we show in Corollary 4.9 under certain assumptions the range of a function $F(\kappa)=\operatorname{cov}\left(\mathcal{M}_{\kappa}\right)$ has at most two elements. The same situation is in the models from Theorems 5.4, 5.5 and 5.7. The problem is how to construct a model in which the range of the function $F$ has three elements.

Question. Is it consistent with ZFC that $\operatorname{cov}(\mathcal{M})=\omega_{4}=\mathfrak{c} \& \operatorname{cov}\left(\mathcal{M}_{\omega_{1}}\right)=\omega_{3} \quad \&$ $\left(\forall \kappa \geq \omega_{2}\right) \operatorname{cov}\left(\mathcal{M}_{\kappa}\right)=\omega_{2} ?$ 


\section{REFERENCES}

[1]. J. Cichoń, handwritten notes, 1986.

[2]. J. Cichoń, On two-cardinal properties of ideals, Trans. Amer. Math. Soc. 314 (1989), 693708.

[3]. J. Cichon,, J. Kraszewski, On some new ideals on the Cantor and Baire spaces, Proc. Amer. Math. Soc. 126 (1998), 1549-1555.

[4]. R. Engelking, General Topology, PWN, 2nd ed., Warsaw, 1985.

[5]. D. H. Fremlin, Measure algebras, Handbook of Boolean Algebras, ed. by J. D. Monk with R. Bonnet, North-Holland, Amsterdam (1989), 879-980.

[6]. D. H. Fremlin, Real-valued measurable cardinals, Israel Math. Conf. Proc. 6 (1993), 151-304.

[7]. S. Fuchino, S. Shelah, L. Soukup, Sticks and clubs, Ann. Pure Appl. Logic 90 (1997), 57-77.

[8]. T. Jech, Multiple forcing, Cambridge University Press, Cambridge, 1986.

[9]. T. Jech, K. Prikry, Ideals over uncountable sets: Application of almost disjoint functions and generic ultrapower, Mem. Amer. Math. Soc. 18, 214 (1979).

[10]. T. Jech, K. Prikry, Cofinality of the partial ordering of functions from $\omega_{1}$ into $\omega$ under eventual domination, Math. Proc. Cambridge Phil. Soc. 95 (1984), 25-32.

[11]. A. Kamburelis, B. We,glorz, Splittings, Arch. Math. Logic 35 (1996), 263-277.

[12]. K. Kunen, Set Theory, North-Holland, Amsterdam, 1980.

[13]. K. Kunen, Random and Cohen reals, Handbook of Set-theoretical Topology, ed. by K. Kunen and J. A. Vaughan, North-Holland, Amsterdam (1984), 889-911.

[14]. A. W. Miller, Some properties of measure and category, Trans. Amer. Math. Soc. 266 (1981), 93-114.

[15]. A. W. Miller, The Baire category theorem and cardinals of countable cofinality, J. Symb. Logic 47 (1982), 275-288.

[16]. J. E. Vaughan, Small uncountable cardinals and Topology, Open Problems in Topology, ed. by J. van Mill and G. M. Reed, North-Holland (1990), 195-218.

Institute of Mathematics, University of Wroclaw, Pl. Grunwaldzki 2/4, 50-156

Wroclaw, POLAND

E-mail address: kraszew@math.uni.wroc.pl 